\title{
A phase $2 / 3$ randomized clinical trial followed by an open-label extension to evaluate the effectiveness of elamipretide in Barth syndrome, a genetic disorder of mitochondrial cardiolipin metabolism
}

\author{
W. Reid Thompson, MD¹, Brittany Hornby, PT, DPT'2, Ryan Manuel, BS³, Elena Bradley, PT, DPT ${ }^{2}$, \\ Janice Laux, $\mathrm{PT}^{2}$, Jim Carr, PharmD ${ }^{4}$ and Hilary J. Vernon, MD, PhD (i) ${ }^{3}$
}

Purpose: To evaluate effectiveness of elamipretide in Barth syndrome (BTHS), a genetic condition of defects in TAZ, which causes abnormal cardiolipin on the inner mitochondrial membrane.

Methods: We performed a randomized, double-blind, placebocontrolled crossover trial followed by an open-label extension in BTHS to test the effect of elamipretide, a mitochondrial tetrapeptide that interacts with cardiolipin. In part 1,12 subjects were randomized to $40 \mathrm{mg}$ per day of elamipretide or placebo for 12 weeks, followed by a 4 -week washout and then 12 weeks on the opposite arm. Ten subjects continued on the open-label extension (part 2) of $40 \mathrm{mg}$ per day of elamipretide, with eight subjects reaching 36 weeks. Primary endpoints were improvement on the 6-minute walk test (6MWT) and improvement on a BTHS Symptom Assessment (BTHS-SA) scale.
Results: In part 1 neither primary endpoint was met. At 36 weeks in part 2, there were significant improvements in 6MWT $(+95.9 \mathrm{~m}, p=0.024)$ and BTHS-SA $(-2.1$ points, $p=$ $0.031)$. There were also significant improvements in secondary endpoints including knee extensor strength, patient global impression of symptoms, and some cardiac parameters.

Conclusion: In this interventional clinical trial in BTHS, daily administration of elamipretide led to improvement in BTHS symptoms.

Genetics in Medicine (2021) 23:471-478; https://doi.org/10.1038/s41436020-01006-8

Keywords: Barth syndrome; elamipretide; cardiolipin; 6-minute walk test

\section{INTRODUCTION}

Barth syndrome (BTHS, OMIM 302060) is a rare X-linked disorder with a prevalence of approximately 1 in $1,000,000$ males. ${ }^{1}$ BTHS is clinically characterized by cardiac left ventricular noncompaction, early onset cardiomyopathy, intermittent neutropenia, abnormal growth, and skeletal myopathy among other features. ${ }^{2}$ The burden of morbidity and mortality is high, and there are currently no disease-specific treatments. ${ }^{2,3}$

BTHS is caused by defects in Tafazzin (TAZ), which encodes for a transacylase involved in the final remodeling step of cardiolipin (CL). ${ }^{3-5} \mathrm{CL}$, located in the inner mitochondrial membrane (IMM), has central roles in mitochondrial maintenance and function including organizing respiratory chain supercomplexes, maintenance of cristae structure, and signaling in apoptosis via interaction with cytochrome c. ${ }^{6-12}$ TAZ deficiency results in abnormal CL content, including an accumulation of monolysocardiolipin (MLCL) and reduction of CL, with resultant mitochondrial dysfunction. ${ }^{6-14}$
SS-31 (elamipretide, D-Arg-2' $6^{\prime}$-dimethylTyr-Lys-Phe$\mathrm{NH}_{2}$ ) is a cell-permeable peptide with an alternating aromatic-cationic structure. SS-31 has been shown to target to the IMM. There, it binds to cardiolipin via electrostatic and hydrophobic interactions, ${ }^{15}$ and is hypothesized to sustain the cristae network and remediate bioenergetic dysfunction. ${ }^{16}$ SS31 has shown benefit in preclinical mitochondrial disorders and heart failure. ${ }^{15,17-20}$

We performed a randomized, double-blind, placebocontrolled crossover trial with an open-label extension to test the clinical efficacy and safety of daily administration of elamipretide in individuals with genetically confirmed Barth syndrome.

\section{Trial design}

\section{MATERIALS AND METHODS}

The "Phase 2 randomized, double-blind, placebo-controlled crossover trial to evaluate the safety, tolerability, and efficacy of subcutaneous injections of elamipretide (MTP-131) in

\footnotetext{
${ }^{1}$ Department of Pediatric Cardiology, Johns Hopkins University School of Medicine, Baltimore, MD, USA; ${ }^{2}$ Department of Physical Therapy, Kennedy Krieger Institute, Baltimore, MD, USA; ${ }^{3}$ Department of Genetic Medicine, Johns Hopkins University School of Medicine, Baltimore, MD, USA; ${ }^{4}$ Stealth BioTherapeutics Inc, Newton, MA, USA.

Correspondence: Hilary J. Vernon (hvernon1@jhmi.edu)

These authors contributed equally: W. Reid Thompson, Brittany Hornby
} 
subjects with genetically confirmed Barth syndrome followed by an open-label treatment extension (SPIBA 201)" (Clinicaltrials.gov NCT03098797) study was a sponsor-initiated, single-site, randomized, blinded, placebo-controlled crossover trial that was conducted at the Johns Hopkins Hospital. Enrollment began July 2017, and the open-label extension is ongoing as of September 2020. The trial design and baseline characteristics of the patients are described below. The trial was supported by Stealth Biotherapeutics, Inc., who designed the trial in conjunction with the Johns Hopkins University study team. The study sponsor was not involved with data collection. The study sponsor was involved with data analysis and the preparation of this publication. The full study protocol can be found in the Supplementary materials.

\section{Ethics approval}

The trial protocol was approved by the Johns Hopkins University School of Medicine Investigational Review Board. The trial was performed in accordance with the principles of the Declaration of Helsinki and with the standards of good clinical practice. Written informed consent was obtained from patients and/or their parent/guardian. Clinicaltrials.gov registration number NCT03098797.

\section{Patient population}

Patients with a molecular confirmation of Barth syndrome, as defined by a pathogenic genetic variant in the TAZ gene, who were 12 years of age and older were eligible for study enrollment. The age of 12 years was selected based on prior nonclinical pharmacokinetic and pharmacodynamic studies. $T A Z$ variants can be found in Supplementary Table 1. Other inclusion criteria included body weight $>30 \mathrm{~kg}$ AND estimated glomerular filtration rate $(\mathrm{eGFR}) \geq 90 \mathrm{~mL} / \mathrm{min} / 1.73 \mathrm{~m}^{2}$ at the screening visit or body weight $>40 \mathrm{~kg}$ AND eGFR $\geq 60 \mathrm{~mL} / \mathrm{min} /$ $1.73 \mathrm{~m}^{2}$ at enrollment. All study participants were male, as Barth syndrome is an X-linked disorder that almost always only affects males. Participants were ambulatory and able to complete a 6-minute walk test. All patients were on stable medications for at least 30 days prior to the study visit. Patients were excluded if they had been hospitalized within 30 days, had uncontrolled hypertension, a history of heart transplantation, or implantation of a cardioverter defibrillator within 3 months or expected implantation. A complete list of the inclusion and exclusion criteria is provided in Supplementary Table 2.

\section{Trial procedures}

For part 1, subjects were randomized (1:1) to one of two sequence groups: 12 weeks of single daily subcutaneous doses of $40 \mathrm{mg}$ elamipretide in treatment period 1 followed by 12 weeks of treatment with placebo in treatment period 2 (separated by 4 -week washout period), or vice versa. Randomization was done via an independent data management team; the study team, participant, and sponsor were blinded to the cohort assignment. During part 1, elamipretide and placebo were supplied in identical appearing packaging and coded by a numeric identifier.
Part 2, an open-label extension study, was available to all participants who completed part 1 . Patients received a daily subcutaneous dose of $40 \mathrm{mg}$ elamipretide for a total drug exposure of 36 weeks. The participant flowsheet is presented in Fig. 1. A graphical schematic of the study design is presented in Supplementary Fig. 3.

\section{Outcome measures}

For part 1, the primary outcome measures were to evaluate the effects of elamipretide administered for 12 weeks on the distance walked in meters during the 6-minute walk test $(6 \mathrm{MWT})$ and on the total fatigue score on the Barth Syndrome Symptom Assessment (BTHS-SA), a scale that measures Barth syndrome-specific symptoms (full scale can be found in Supplementary Figs. 1 and 2). ${ }^{21}$ Secondary outcome measures included knee extensor muscle strength as measured by handheld dynamometry (HHD), ${ }^{22}$ five times sitto-stand test (5XSST), SWAY balance measurements, ${ }^{23,24}$ two and three dimensional (2D and 3D) echocardiographic measurements, patient reported outcomes (PROs), Clinician Global Impression (CGI) scales, ${ }^{25}$ Caregiver Global Impression (CaGI) scales, and other measures. A full list of secondary outcomes can be found in the Supplementary Appendix Table 3. All assessments were administered and scored in accordance with standardized test procedures.

For part 2, the primary outcome was to assess the long-term safety and tolerability of daily elamipretide. Secondary outcome measures included 6MWT, total fatigue score on the BTHS-SA, knee extensor muscle strength as measured by HHD, 5XSST, 2D and 3D echocardiographic measurements, patient reported outcomes, CGI scales, CaGI scales, and other measures. A full list of secondary outcomes can be found in the Supplementary Appendix Table 3.

\section{Statistical analysis}

For this phase 2, randomized, double-blind, placebo-controlled crossover trial followed by an open-label treatment extension assessment, a sample size of approximately 12 subjects was planned. Assuming an underlying standard deviation of paired differences of $50 \mathrm{~m}$ for the $6 \mathrm{MWT}$ distance and 1.3 points for the BTHS-SA total fatigue score, 12 subjects provide for nearly $80 \%$ power to detect a mean improvement of $50 \mathrm{~m}$ in the $6 \mathrm{MWT}$ or 1.3 points for the BTHS-SA total fatigue score, with each potentially tested at the 0.025 (two-sided) level of significance (associated with a potential adjustment via Hochberg's procedure) in part 1. Two primary endpoints were included in the primary endpoint family (6MWT and BTHS-SA total fatigue). Hochberg's procedure was used to control the family-wise type I error rate at 0.05 (two-sided). No adjustments were made to alpha levels to account for secondary efficacy measures.

\section{RESULTS}

\section{Patient characteristics}

A total of 16 subjects were screened and 12 (75.0\%) of these subjects were randomized into part 1 of the study. Reasons for 


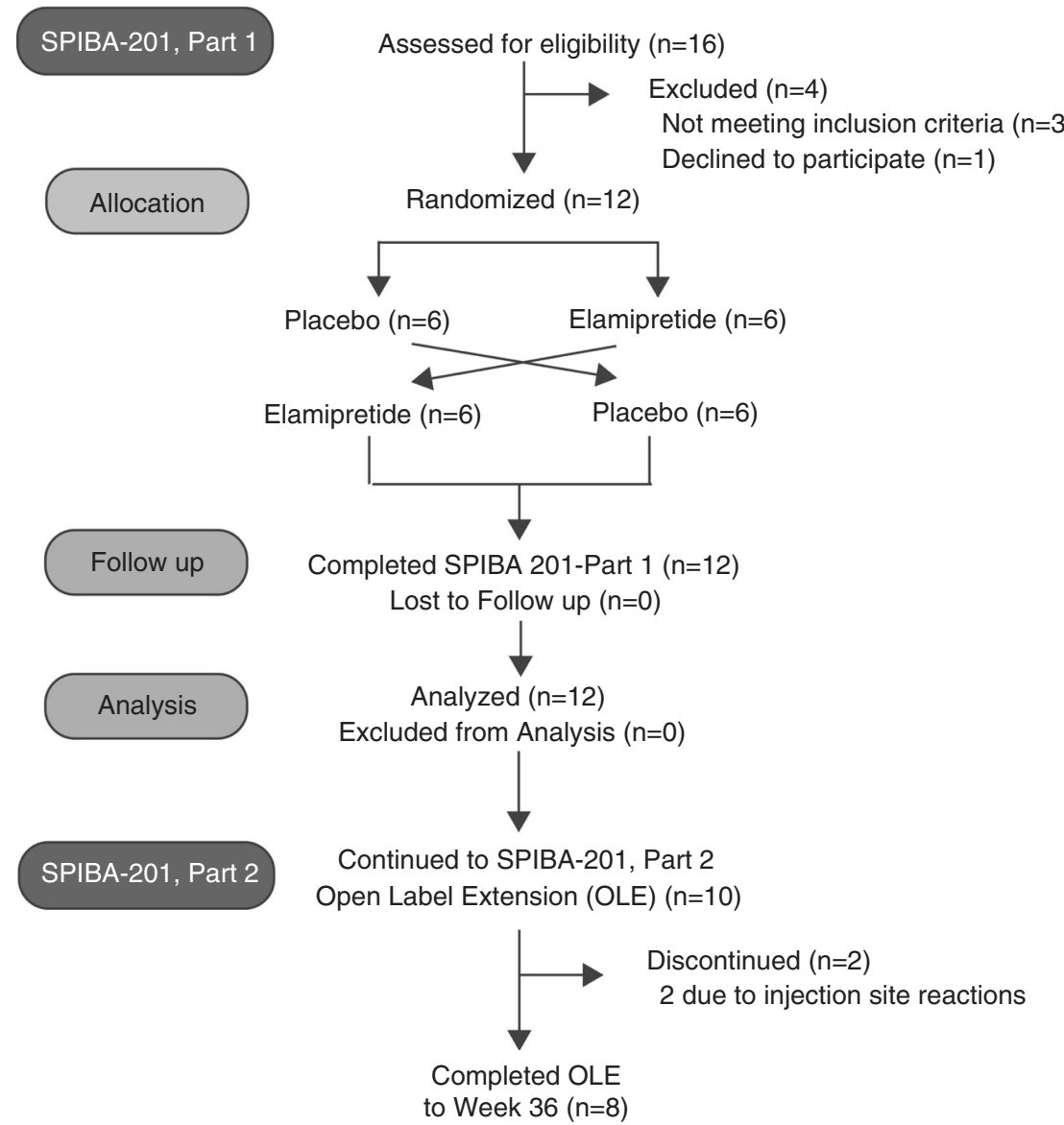

Fig. 1 Study participant flowsheet for SPIBA 201 to test the efficacy and safety of single daily subcutaneous (SC) doses of 40 mg elamipretide (vs. placebo) in subjects with Barth syndrome. Subjects were randomized (1:1) to one of two sequence groups: 12 weeks of single daily SC doses of 40 mg elamipretide in treatment period 1 followed by 12 weeks of treatment with placebo in treatment period 2 (separated by 4-week washout period), or vice versa. The crossover design was selected because of the additional anticipated power of subjects serving as their own control, given the anticipated scarcity of subjects available to participate in this study due to the ultrarare nature of Barth syndrome.

screen failure included recent or anticipated changes in medications $(n=3)$ and patient declining to enroll due to anticipated difficulty completing the study activities $(n=1)$. All participants had a pathogenic variant identified in the TAZ gene (Supplementary Table 1). Average age at enrollment was 19.5 years (range 12-35 years), all enrollees were male, 11 participants identified as white and one identified as multiracial. At baseline, 2 of 12 individuals had neutropenia as defined by an absolute neutrophil count $<1500$ cells $/ \mu \mathrm{L}$, and 6 individuals received granulocyte colony-stimulating factor (G-CSF) at baseline. Mean distance walked on the 6MWT was 395.5.m $(+/-59.9 \mathrm{SD})$, and mean BTHS-SA total fatigue score was 8.0 $(+/-1.3 \mathrm{SD})$ at enrollment. Detailed baseline characteristics are described in Table $\mathbf{1}$ and characteristics of each of the randomized cohorts in part 1 (Supplementary Table 4).

Outcomes and follow-up: part 1-placebo-controlled study All 12 randomized subjects completed both treatment periods in part 1 . After 12 weeks of elamipretide therapy in part 1 , a statistical difference was not observed in the distance walked on the 6MWT compared with placebo $(-0.8 \mathrm{~m}, p=0.97)$. After 12 weeks of elamipretide therapy in part 1, statistical difference was also not observed in the BTHS-SA total fatigue score compared with placebo $(+0.06 ; p=0.89)$. A sequence effect was not observed for either primary endpoint. After 12 weeks of elamipretide therapy in part 1, statistical differences were not observed in muscle strength by HHD compared with placebo ( +6.7 newtons; $p=0.65)$ or any of the other secondary endpoints.

\section{Outcomes and follow-up: part 2-open-label extension}

Ten participants continued to part 2. Two of the ten participants discontinued the study during part 2 due to injection site reactions at week 12, part 2. Follow-up data for all outcomes were available through week 36 , part 2 in eight participants. No patients were lost to follow-up (Fig. 1).

The mean distance walked on the 6MWT increased from baseline across ten subjects at week 12 , part 2 , with a mean improvement of $60.5 \mathrm{~m}$ (16\% increase, paired $t$-test: $p=0.02)$. At week 36, part 2 across eight subjects the mean improvement from baseline was $95.9 \mathrm{~m}$ (25\% improvement, paired $t$-test: $p=0.02$ ) (Fig. 3a).

Decreases in the mean BTHS-SA total fatigue score from baseline were observed for all ten subjects at week 12, part 2, 
Table 1 Baseline characteristics of enrolled patients. Demographic variable Demographic result

\begin{tabular}{|c|c|}
\hline Mean age (years) (range) & $19.5(12-35)$ \\
\hline Male $(n)$ & 12 \\
\hline \multicolumn{2}{|l|}{ Race $(n)$} \\
\hline White & 11 \\
\hline Multiracial & 1 \\
\hline \multicolumn{2}{|l|}{ Ethnicity $(n)$} \\
\hline Not Hispanic or Latino & 12 \\
\hline Hispanic or Latino & 0 \\
\hline Mean height $(\mathrm{cm})$ & $167.3(150.4-187.7)$ \\
\hline Mean weight $(\mathrm{kg})$ & $50.8(31.4-85.9)$ \\
\hline $\mathrm{BMI}\left(\mathrm{kg} / \mathrm{m}^{2}\right)$ & $17.6(13.6-24.4)$ \\
\hline Mean 6MWT (meters) & 395.5 \\
\hline Mean BTHS-SA total fatigue & 8 \\
\hline Mean 3D LV end-diastolic volume (Z-score [SD]) & $-2(1.34)$ \\
\hline Mean 3D LV stroke volume (Z-score [SD]) & $-1.84(1.53)$ \\
\hline Mean ejection fraction (\% [SD]) & $60.6(4.0)$ \\
\hline \multicolumn{2}{|c|}{$\begin{array}{l}\text { All } 12 \text { randomized subjects completed both treatment periods in part 1. Overall, the } \\
\text { majority of subjects in part } 1 \text { were White (91.7\%), Non-Hispanic or Latino (100.0\%), } \\
\text { with a mean (SD) age of } 19.5(7.65) \text { years. Mean (SD) weight and BMI were } 50.84 \\
\text { (18.934) and } 17.56(3.818) \text {, respectively. One subject (16.7\%) included in the trial } \\
\text { was Indian or an Alaskan Native. Sequence AB (elamipretide-placebo) were slightly } \\
\text { older, ranging in age between } 14 \text { and } 35 \text { years (mean [SD]: } 23.3 \text { [9.07] years), while } \\
\text { subjects in treatment sequence BA (placebo-elamipretide) were slightly younger, } \\
\text { ranging in age between } 12 \text { and } 21 \text { years (mean [SD]: } 15.7 \text { [3.33] years), compared } \\
\text { with the overall population, which ranged between } 12 \text { and } 35 \text { years. For subjects in } \\
\text { treatment sequence AB, mean weight and BMl were } 54.50 \mathrm{~kg} \text { and } 18.23 \mathrm{~kg} / \mathrm{m}^{2} \text {, } \\
\text { respectively. Mean weight and BMl were lower for subjects in treatment sequence } \\
\text { BA (47.18 kg and } 16.88 \mathrm{~kg} / \mathrm{m}^{2} \text {, respectively). Demographics of each treatment } \\
\text { sequence were generally similar to the overall population with some variability } \\
\text { observed in overall age, age ranges, weight, and BMI. } \\
6 M W T \text { 6-minute walk test, BTHS-SA Barth Syndrome Symptom Assessment scale, } \\
\text { BMl body mass index, } L V \text { left ventricular. }\end{array}$} \\
\hline
\end{tabular}

with mean improvement of -1.6 points (average $19 \%$ reduction/improvement, paired $t$-test: $p=0.03$ ) Decreases in the mean BTHS-SA total fatigue score from baseline were observed for all eight subjects at week 36 , part 2 with a mean improvement from baseline of -2.1 points (26\% reduction/ improvement) (paired $t$-test: $p=0.03$ ).

At week 12, part 2 there was a mean improvement from baseline HHD of 37.9 newtons (30\% improvement, paired $t$ test: $p=0.003$ ) across the ten subjects. At week 36, part 2 there was a mean improvement of 56.0 newtons (42\% improvement, paired t-test: $p=0.001)$ across the eight participants (Fig. 3b).

Decreases (improvements) in the mean Clinician Global Impression (CGI) of symptom severity from baseline were observed for all ten subjects at week 12 part 2, although statistical significance was not achieved, with a mean improvement of -0.2 points (a $15 \%$ reduction, paired $t$-test: $p=0.17)$. For the cohort of eight subjects who reached week 36 , part 2 , the mean improvement was -0.6 points $(43 \%$ reduction, paired $t$-test: $p=0.10)$ from baseline. Decreases (improvements) in the mean Patient Global Impression (PGI) of Symptoms severity from baseline were observed for all ten subjects at week, 12 part 2, although statistical significance was not achieved, with a mean improvement of -0.4 points (a $21 \%$ reduction, paired $t$-test: $p=0.17)$. For the cohort of eight subjects who reached week 36 , part 2 , the mean improvement was -1.2 points (35\% reduction, paired $t$-test: $p=0.05$ ) from baseline.

Decreases in the mean PROMIS Fatigue Short Form T-score were observed in ten subjects at week 12, part 2 with a mean improvement of -6.0 from baseline (an 11\% reduction, paired

\section{SPIBA-201 Part 2 Week 36 Summary of Treatment Effect Change from Baseline $\mathrm{N}=8$}

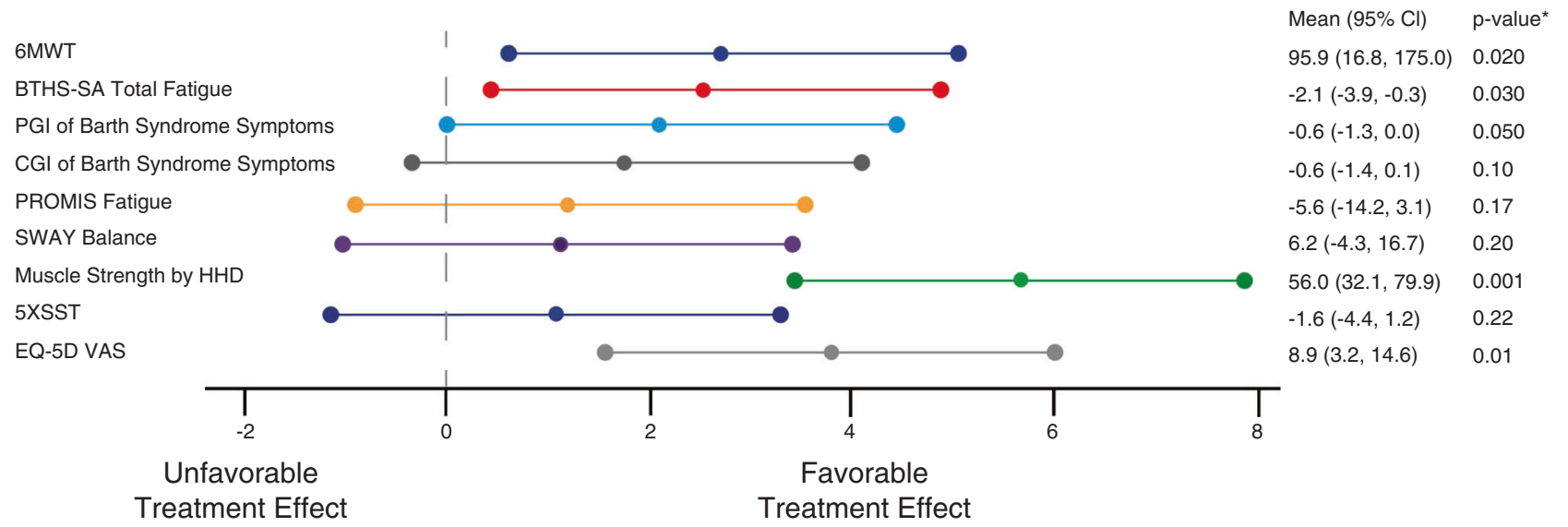

Fig. 2 Outcome measures at week 36, part $\mathbf{2}$ in $\mathbf{8}$ patients. Statistical significance was achieved in improved measures for 6 -minute walk test (m), the Barth Syndrome Symptom Assessment (BTHS-SA) total fatigue scale (mean score), Patient Global Impression (PGI) of symptoms (mean score), muscle strength measured by handheld dynamometry (HHD) (newtons), and the EQ-5D questionnaire Fig. 2. Favorable treatment effects were also seen in other measures (Promis Fatigue [mean score], Clinician Global Impression [CGI] of Barth syndrome symptoms [mean score], and SWAY balance), though not achieving statistical significance. Treatment effect was rescaled to a T-score and standard error unit of $1 .{ }^{*} p$ values represent a $t$-test for matched pairs, comparing mean at baseline to mean at week 36 in part 2. 

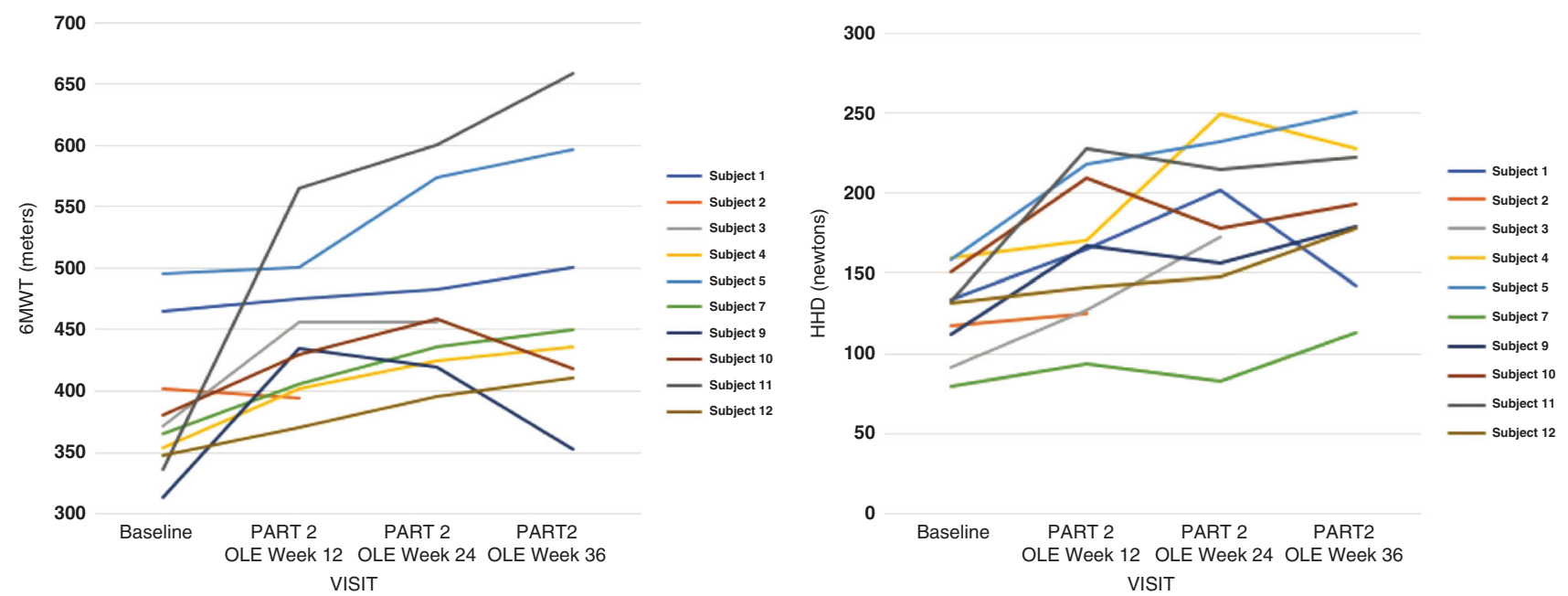

Fig. 3 6-minute walk test and hand held dynamometry results per participant from baseline to open label extension, week 36. (a) Change in 6-minute walk test (6MWT) results per participant from baseline to open label extension (OLE) week 36. The mean distance walked on the 6MWT increased from baseline across ten subjects at week 12, part 2, with a mean improvement of $60.5 \mathrm{~m}$ (16\% increase, paired t-test: $p=0.02)$. At week 36, part 2 across eight subjects the mean improvement from baseline was $95.9 \mathrm{~m}$ (25\% improvement, paired $t$-test: $p=0.02)$. (b) Change in handheld dynamometry (HHD) results per participant from baseline to open label extension (OLE) week 36. At week 12, part 2 there was a mean improvement from baseline HHD of 37.9 newtons (a 30\% improvement, paired $t$-test: $p=0.003$ ) across the ten subjects. At week 36 , part 2 there was a mean improvement of +56.0 newtons (a $42 \%$ improvement, paired $t$-test: $p=0.001$ ) across the eight participants who reached the 36 week study visit.

$t$-test: $p=0.05)$. All eight subjects who reached week 36, part 2 had an improvement in the PROMIS score, with a mean improvement of -5.6 points though statistical significance was not achieved (a $10 \%$ reduction, paired $t$-test: $p=0.17$ ).

While there were trends toward improvement for the SWAY balance across the ten subjects at week 12, part 2 and the eight subjects at week 36 , part 2 , statistical significance was not achieved. Similar trends were seen for 5XSST.

The $2 \mathrm{D}$ and $3 \mathrm{D}$ echocardiogram measurements were compared between elamipretide and placebo at 12 weeks, and between baseline and week 36, part 2 (for a full profile of echocardiographic changes see Supplementary Table 5). Treatment with elamipretide resulted in a $16 \%$ improvement in average left ventricular stroke volume indexed to body surface area (BSA), from baseline $\left(30.5 \mathrm{~mL} / \mathrm{m}^{2}\right)$ to week 36 $\left(35.3 \mathrm{~mL} / \mathrm{m}^{2}\right)$ of part 2. Utilizing a slope model of individual regression lines for each subject to determine the consistent change in indexed stroke volume over the course of exposure, there was a significant trend of an increase in stroke volume over time $(p<0.01)$ Supplementary Fig. 4 . For the eight subjects who completed week 36 , part 2 mean stroke volume $z$-scores increased from -1.38 at baseline to -0.56 at week 36 , part 2, respectively. Left ventricular end-diastolic volume and left ventricular end-systolic volume indexed to BSA trends over time can be found in Supplementary Figs. 5 and 6 .

\section{Other outcomes}

Throughout the course of study participation, 3/12 participants had at least one absolute neutrophil count $<500$ cells/ $\mu \mathrm{L}, 5 / 12$ participants had at least one absolute neutrophil count $<1000$ cells $/ \mu \mathrm{L}$, and $9 / 12$ participants had at least one absolute neutrophil count $<1500$ cells $/ \mu \mathrm{L}$. There was no statistically significant change in neutrophil counts between placebo and elamipretide in part 1 or between baseline and week 36, part 2 of the study. There was no statistically significant change in FGF21, GDF15, bloodspot monolysocardiolipin to cardiolipin ratio, or 3-methyglutaconic acid levels in urine or plasma, between placebo and elamipretide in part 1, or between baseline and week 36 , part 2 of the study.

\section{Safety}

In part 1, there were 121 reported treatment-emergent adverse events (TEAEs); 74 events were reported in the elamipretide group and 47 events were reported in the placebo group (Supplementary Table 6). Twelve of 12 (100\%) participants receiving elamipretide had at least one TEAE and 10/12 (83.3\%) participants receiving placebo had at least one TEAE (Table 2). The majority of events were injection site reactions, including injection site erythema (12/12 elamipretide vs. $3 / 12$ placebo), injection site pain $(9 / 12$ elamipretide vs. $4 / 12$ placebo), injection site induration (8/12 elamipretide vs. $2 / 12$ placebo). Other TEAEs included bronchitis (2/12 elamipretide vs. $1 / 12$ placebo), headache (1/12 elamipretide vs. 3/12 placebo) (Table 2). Most events were mild to moderate in severity. There was one serious TEAE deemed not to be related to elamipretide (hospitalization for costochondritis).

By week 36, part 2,100\% of participants had at least one TEAE, most of which were mild to moderate in severity (Table 2 and Supplementary Table 7). The majority of events were injection site reactions, including but not limited to injection site erythema (8/10), injection site pain $(7 / 10)$, and injection site pruritis $(7 / 10)$. Other TEAEs included dizziness $(4 / 10)$ and headache (3/10). (Table 2). 
Table 2 Treatment-emergent adverse events (TEAEs) in part 1 and part 2, open label extension.

\begin{tabular}{|c|c|c|}
\hline SPIBA 201, part 1 & $\begin{array}{l}\text { Elamipretide } \\
40 \mathrm{mg}, \\
N=12(\%)\end{array}$ & $\begin{array}{l}\text { Placebo } \\
N=12(\%)\end{array}$ \\
\hline At least one TEAE & $12(100.0)$ & $10(83.3)$ \\
\hline $\begin{array}{l}\text { General disorders and administration } \\
\text { site conditions }\end{array}$ & $12(100.0)$ & $8(66.7)$ \\
\hline Injection site erythema & $12(100.0)$ & $3(25.0)$ \\
\hline Injection site pain & $9(75.0)$ & $4(33.3)$ \\
\hline Injection site induration & $8(66.7)$ & $2(16.7)$ \\
\hline Injection site pruritus & $8(66.7)$ & $2(16.7)$ \\
\hline Injection site bruising & $3(25.0)$ & 0 \\
\hline Injection site urticaria & $3(25.0)$ & 0 \\
\hline Medical device site erythema & $1(8.3)$ & $1(8.3)$ \\
\hline Medical device site irritation & $2(16.7)$ & $1(8.3)$ \\
\hline Nervous system disorders & $3(25.0)$ & $5(41.7)$ \\
\hline Headache & $1(8.3)$ & $3(25.0)$ \\
\hline Dizziness & $1(8.3)$ & $1(8.3)$ \\
\hline Gastrointestinal disorders & $1(8.3)$ & $4(33.3)$ \\
\hline Aphthous ulcer & 0 & $2(16.7)$ \\
\hline Nausea & $1(8.3)$ & $1(8.3)$ \\
\hline Infections and infestations & 4 (33.3) & $3(25.0)$ \\
\hline Bronchitis & $2(16.7)$ & $1(8.3)$ \\
\hline Viral upper respiratory tract infection & $1(8.3)$ & $2(16.7)$ \\
\hline Pharyngitis streptococcal & $1(8.3)$ & $2(16.7)$ \\
\hline Sinusitis & $1(8.3)$ & $1(8.3)$ \\
\hline $\begin{array}{l}\text { Injury, poisoning, and procedural } \\
\text { complications }\end{array}$ & $2(16.7)$ & $3(25.0)$ \\
\hline Ligament sprain & $2(16.7)$ & $1(8.3)$ \\
\hline Fall & $1(8.3)$ & $1(8.3)$ \\
\hline
\end{tabular}

SPIBA 201, part 2 open-label extension

Elamipretide $40 \mathrm{mg}$, $N=10(\%)$

\begin{tabular}{ll}
\hline At least one TEAE & $\mathbf{1 0 ( 1 0 0 . 0 )}$ \\
$\begin{array}{l}\text { General disorders and administration site } \\
\text { conditions }\end{array}$ & $\mathbf{1 0 ( 1 0 0 . 0 )}$ \\
Injection site erythema & $8(80.0)$ \\
Injection site pain & $7(70.0)$ \\
Injection site pruritus & $7(70.0)$ \\
Nervous system disorders & $\mathbf{6}(\mathbf{6 0 . 0 )}$ \\
Dizziness & $4(40.0)$ \\
Injury, poisoning, and procedural complications & $\mathbf{4}(\mathbf{4 0 . 0 )}$ \\
Joint dislocation & $2(20.0)$ \\
Respiratory, thoracic and mediastinal disorders & $\mathbf{3}(\mathbf{3 0 . 0 )}$ \\
Oropharyngeal pain & $2(20.0)$ \\
\hline
\end{tabular}

In part 1, at least one TEAE was reported in $100 \%(12 / 12)$ of subjects receiving $40 \mathrm{mg}$ elamipretide and in $83 \%(10 / 2)$ of subjects receiving placebo. Treatmentrelated TEAEs occurred more often in subjects when receiving $40 \mathrm{mg}$ elamipretide (48 events in 12 [100.0\%] subjects) compared with when receiving placebo (13 events in 8 [66.7\%] subjects); all of the treatment-related TEAEs on elamipretide are injection site related. The most common TEAE overall was injection site erythema, followed by injection site pain, with higher incidence of subjects treated with elamipretide $(100.0 \%$ and $75.0 \%$, respectively) compared with placebo $(25.0 \%$ and $33.3 \%$, respectively). The majority of TEAEs were mild or moderate in severity. There were more TEAEs that were moderate in severity in subjects treated with elamipretide (6 [50.0\%] subjects) than with placebo (1 [8.0\%] subjects). There were no subjects with severe TEAEs reported. By week 36, part 2 there were 129 TEAEs reported in $10(100 \%)$ subjects and 45 treatment-related TEAEs reported in ten subjects $(100 \%)$. There were two subjects with TEAEs leading to discontinuation of study treatment. The most common TEAEs were injection site erythema followed by injection site pain/pruritus, and dizziness. The majority of TEAEs were mild or moderate in severity. System organ classes (SOC) are bolded in the first column, and $N(\%)$ represents the total number of subjects (of ten total) who experienced a TEAE within that SOC. Treatment-emergent adverse event data shown are $N(\%)$ of total patients. subjects who experienced more than one TEAE within an individual SOC were counted only once (thus in some SOCs, the $N[\%]$ may be less than the sum of the $N[\%]$ of the subheading TEAEs). A TEAE is listed in this table if it was experienced by two or more subjects, but may be included in the number of subjects who experienced an event corresponding to a given SOC (thus in some SOCs, the $N$ [\%] may be greater than the sum of the $N[\%]$ of the subheading TEAEs). Only the worst grade of severity is counted for multiple occurrences of the same adverse event for a given subject.

\section{DISCUSSION}

Barth syndrome is an ultrarare genetic condition for which there are currently no disease-specific therapies. Here we present data supporting the beneficial effects of elamipretide in the skeletal muscle symptoms of disease, with other possible beneficial effects in cardiac stroke volume.

After 12 weeks of elamipretide exposure, we did not see significant improvements in primary or secondary outcomes compared with placebo. However, in part 2 of the study, after 48 total weeks of drug exposure (combined exposure in part 1 and part 2), we observed increasing and persistent improvements in multiple domains across the study population. This delayed effect may be because it takes a significant period of time for skeletal and cardiac muscle remodeling to occur prior to observing measurable benefit. ${ }^{26}$ We have previously shown that 6MWT distance and lower extremity muscle strength do not improve over time in individuals with BTHS, and in fact, may worsen with age. ${ }^{23,27}$ Therefore, while there is the potential for a placebo effect contributing to the benefits in skeletal muscle endurance and strength observed in part 2 , we believe that the lasting and significant improvement combined with the deviation from the expected natural history of disease, makes this finding likely to be an elamipretide-related physiologic improvement. There are currently no other known interventions to significantly improve skeletal muscle endurance and lower extremity strength in individuals with BTHS. Endurance training in individuals with other mitochondrial disorders has been shown to significantly increase maximal oxygen uptake and peripheral muscle strength, ${ }^{28}$ but this has not been demonstrated in Barth syndrome. ${ }^{29}$ In a small group of individuals with BTHS $(n=3)$, a supervised, 12-week progressive resistance exercise program resulted in significant improvements in upper extremity muscle strength but not in lower extremity (knee extension and knee flexion) strength, torque, or power. ${ }^{30}$

There was some variation in improvement in distance walked within individual patients from time point to time point; for example patients 9 and 10 had a decrease in 6MWT distance between open-label extension 24 weeks and openlabel extension 36 weeks, though both were still above baseline, and subjects 1 and 4 had a decrease in strength testing on hand held dynamometry between open-label extension 24 weeks and open-label extension 36 weeks, though subject 4 was still above baseline. This may represent some variability inherent in effort-dependent outcomes or possible intercurrent extenuating circumstances. It is notable that these decreases between weeks 24 and 36 weeks openlabel extension were not seen across all outcome measures in the individual participants.

Interestingly, we did observe increases in cardiac stroke volume in the study population. It is noteworthy that at baseline, while none of the study participants had abnormal left ventricular ejection fraction, average end-diastolic volume was 2 SD below the mean, with resulting low-normal stroke 
volume. The cardiomyopathy and heart failure seen in BTHS has primarily been associated with left ventricular dilatation and reduced ejection fraction, though apical noncompaction and hypertrophic cardiomyopathy is also seen. ${ }^{31} \mathrm{~A}$ restrictive phenotype with low ventricular volume and preserved ejection fraction has not previously been described in BTHS, but is at least suggested by the baseline data in this study. The increase in left ventricular volumes at 36 weeks of elamipretide treatment may represent remodeling with improvement in myocardial stiffness due to elamipretide. At this time, it is difficult to know whether the increases in stroke volume seen in the treatment group will have a beneficial impact on longterm cardiac outcome or exercise tolerance; however, the open-label extension of this study is ongoing to 168 weeks, and clinical observations potentially beyond this time period could be informative.

In general, elamipretide was well tolerated. The side effects of elamipretide were generally confined to injection site reactions, which were mostly mild in nature but led to two patients discontinuing part 2 of the study.

Given the lack of significant change in MLCL/CL ratio in the setting of clinical benefit with elamipretide, we speculate that elamipretide may have local effects on the mitochondrial defects caused by abnormal CL, such as increased stabilization of disorganized respiratory chain complexes, improvement in cristae organization, or prevention of oxidation of CL and/or MLCL. These mechanism-specific hypotheses merit further investigation.

\section{Limitations}

The significant increases in primary and secondary endpoints were observed in the open-label extension part of the study and lacked a placebo-controlled group. Thus, placebo effect cannot be ruled out as a factor in the BTHS symptom improvement. To strengthen the significance of these findings, we are pursuing a matched natural history comparison cohort to serve as a surrogate placebo control. Encouragingly, the effects on stroke volume are not subject to placebo effect, and support the positive physiological effects of elamipretide in BTHS. Additionally, 6MWT, as a dependent functional test, can be subject to patient learning effects, ${ }^{32}$ although in a systemic literature review 6MWT was shown to have good reliability and significant utility in predicting functional capacity in patients with heart failure who walk less than $490 \mathrm{~m}^{33}$

Additionally, as Barth syndrome is often an infantile-onset disorder, it is important to understand whether earlier administration of elamipretide could have a more profound effect on clinical outcomes. ${ }^{3}$ Due to the small number of study participants, we were unable to perform meaningful analysis of response to elamipretide based on patient age of enrollment, and our lower age limit of participation was 12 years of age. In the future, it will be important to study the possible effects of elamipretide on younger children, with consideration to both age-appropriate outcome measures, safety, and pharmacodynamics.

\section{Conclusion}

In conclusion, in our trial elamipretide utilization for a total of 48 weeks of exposure (12 weeks in part 1 and 36 weeks in part 2) correlated with significant improvements in skeletal muscle performance and patient perception of fatigue and disease symptoms. We also observed increases in cardiac stroke volumes, with possible implications for benefit in the cardiac dysfunction of BTHS. Given these findings, we conclude that elamipretide may have a beneficial role in the ongoing care of individuals with Barth syndrome.

\section{SUPPLEMENTARY INFORMATION}

The online version of this article (https://doi.org/10.1038/s41436020-01006-8) contains supplementary material, which is available to authorized users.

\section{ACKNOWLEDGEMENTS}

This clinical trial was funded by Stealth BioTherapeutics.

\section{DISCLOSURE}

J.C. is employed by Stealth BioTherapeutics of Newton, Massachusetts. The other authors declare no conflicts of interest.

Publisher's note Springer Nature remains neutral with regard to jurisdictional claims in published maps and institutional affiliations.

\section{REFERENCES}

1. Miller PC, Ren M, Schlame M, et al. A Bayesian analysis to determine the prevalence of Barth syndrome in the pediatric population. J Pediatr. 2020;217:139-144.

2. Clarke SL, Bowron A, Gonzalez IL, et al. Barth syndrome. Orphanet J Rare Dis. 2013;8:23.

3. Ferreira C, Pierre G, et al. Barth syndrome. 2014 Oct [Updated $2020 \mathrm{Jul}$ 9]. In: Adam MP, Ardinger HH, Pagon RA, et al., editors. GeneReviews. Seattle, WA: University of Washington; 1993-2020.

4. Bione S, D'Adamo P, Maestrini E, et al. A novel X-linked gene, G4.5. is responsible for Barth syndrome. Nat Genet. 1996;12:385-389.

5. D'Adamo P, Fassone L, Gedeon A, et al. The X-linked gene G4.5 is responsible for different infantile dilated cardiomyopathies. Am J Hum Genet. 1997;61:862-867.

6. Zhang M, Mileykovskaya E, Dowhan W. Gluing the respiratory chain together. Cardiolipin is required for supercomplex formation in the inner mitochondrial membrane. J Biol Chem. 2002;277:43553-43556.

7. Zhang M, Mileykovskaya E, Dowhan W. Cardiolipin is essential for organization of complexes III and IV into a supercomplex in intact yeast mitochondria. J Biol Chem. 2005;280:29403-29408.

8. Claypool SM. Cardiolipin, a critical determinant of mitochondrial carrier protein assembly and function. Biochim Biophys Acta. 2009;1788: 2059-2068.

9. Ostrander DB, Sparagna GC, Amoscato AA, et al. Decreased cardiolipin synthesis corresponds with cytochrome $c$ release in palmitate-induced cardiomyocyte apoptosis. J Biol Chem. 2001;276:38061-38067.

10. Sedlak E, Robinson NC. Phospholipase $A(2)$ digestion of cardiolipin bound to bovine cytochrome c oxidase alters both activity and quaternary structure. Biochemistry. 1999;38:14966-14972.

11. Schwall CT, Greenwood VL, Alder NN. The stability and activity of respiratory Complex II is cardiolipin-dependent. Biochim Biophys Acta. 2012;1817:1588-1596.

12. Pfeiffer K, Gohil V, Stuart RA, et al. Cardiolipin stabilizes respiratory chain supercomplexes. J Biol Chem. 2003;278:52873-52880.

13. $\mathrm{Xu} Y$, Malhotra $A$, Ren $M$, Schlame $M$. The enzymatic function of tafazzin. J Biol Chem. 2006;281:39217-39224.

14. Brandner K, Mick DU, Frazier AE, et al. Taz1, an outer mitochondrial membrane protein, affects stability and assembly of inner membrane protein complexes: implications for Barth syndrome. Mol Biol Cell. 2005;16:5202-5214. 
15. Szeto HH. First-in-class cardiolipin-protective compound as a therapeutic agent to restore mitochondrial bioenergetics. Br J Pharmacol. 2014; 171:2029-2050.

16. Allen ME, Pennington ER, Perry JB, et al. The cardiolipin-binding peptide elamipretide mitigates fragmentation of cristae networks following cardiac ischemia reperfusion in rats. Commun Biol. 2020;3:389.

17. Dai DF, Hsieh EJ, Chen T, et al. Global proteomics and pathway analysis of pressure-overload-induced heart failure and its attenuation by mitochondrial-targeted peptides. Circ Heart Fail. 2013;6: 1067-1076.

18. Birk AV, Liu S, Soong $Y$, et al. The mitochondrial-targeted compound SS-31 re-energizes ischemic mitochondria by interacting with cardiolipin. J Am Soc Nephrol. 2013;24:1250-1261.

19. Dai W, Shi J, Gupta RC, et al. Bendavia, a mitochondria-targeting peptide, improves postinfarction cardiac function, prevents adverse left ventricular remodeling, and restores mitochondria-related gene expression in rats. J Cardiovasc Pharmacol. 2014;64:543-553.

20. Machiraju $P$, Wang $X$, Sabouny $R$, et al. SS-31 peptide reverses the mitochondrial fragmentation present in fibroblasts from patients with DCMA, a mitochondrial cardiomyopathy. Front Cardiovasc Med. 2019;6:167.

21. ATS Committee on Proficiency Standards for Clinical Pulmonary Function Laboratories. ATS statement: guidelines for the six-minute walk test. Am J Respir Crit Care Med. 2002;166:111-117.

22. Avers D, Brown M. Daniels and Worthingham's muscle testing: techniques of manual examination and performance testing. St. Louis, MO: Elsevier 2019.

23. Hornby B, McClellan R, Buckley L, et al. Functional exercise capacity, strength, balance and motion reaction time in Barth syndrome. Orphanet J Rare Dis. 2019;14:37.

24. Patterson JA, Amick RZ, Thummar T, Rogers ME. Validation of measures from the smartphone sway balance application: a pilot study. Int J Sports Phys Ther. 2014:9:135-139.

25. Guy W, Bonato RR, U.S. Department of Health, Education, and Welfare, National Institute of Mental Health. Manual for the ECDEU assessment battery. Chevy Chase, MD: National Institute of Mental Health; 1970.

26. Liu J, Saul D, Boker KO, et al. Current methods for skeletal muscle tissue repair and regeneration. Biomed Res Int. 2018;2018:1984879.
27. Thompson WR, DeCroes B, McClellan $R$, et al. New targets for monitoring and therapy in Barth syndrome. Genet Med. 2016;18: 1001-1010.

28. Parikh S, Goldstein A, Koenig MK, et al. Diagnosis and management of mitochondrial disease: a consensus statement from the Mitochondrial Medicine Society. Genet Med. 2015;17:689-701.

29. Cade WT, Reeds DN, Peterson LR, et al. Endurance exercise training in young adults with Barth syndrome: a pilot study. JIMD Rep. 2017;32:15-24.

30. Bittel AJ, Bohnert KL, Reeds DN, et al. Reduced muscle strength in Barth syndrome may be improved by resistance exercise training: a pilot study. JIMD Rep. 2018;41:63-72.

31. Kang SL, Forsey J, Dudley D, et al. Clinical characteristics and outcomes of cardiomyopathy in Barth syndrome: the UK experience. Pediatr Cardiol. 2016;37:167-176.

32. Wu G, Sanderson B, Bittner V. The 6-minute walk test: how important is the learning effect? Am Heart J. 2003;146:129-133.

33. Pollentier $B$, Irons SL, Benedetto $C M$, et al. Examination of the six minute walk test to determine functional capacity in people with chronic heart failure: a systematic review. Cardiopulm Phys Ther J. 2010;21:13-21.

Open Access This article is licensed under a Creative Commons Attribution-NonCommercial-NoDerivatives 4.0 International License, which permits any non-commercial use, sharing, distribution and reproduction in any medium or format, as long as you give appropriate credit to the original author(s) and the source, and provide a link to the Creative Commons license. You do not have permission under this license to share adapted material derived from this article or parts of it. The images or other third party material in this article are included in the article's Creative Commons license, unless indicated otherwise in a credit line to the material. If material is not included in the article's Creative Commons license and your intended use is not permitted by statutory regulation or exceeds the permitted use, you will need to obtain permission directly from the copyright holder. To view a copy of this license, visit http://creativecommons.org/licenses/by-nc-nd/4.0/.

(c) The Author(s) 2020 